\title{
Wigner molecules in quantum dots
}

\author{
Boris Reusch, ${ }^{1}$ Wolfgang Häusler ${ }^{2}$ and Hermann Grabert ${ }^{1}$ \\ ${ }^{1}$ Fakultät für Physik, Albert-Ludwigs-Universität, D-79104 Freiburg, Germany \\ ${ }^{2}$ I. Institut für Theoretische Physik, Universität Hamburg, D-20355 Hamburg, Germany
}

(Date: November 2, 2018)

We perform unrestricted Hartree-Fock (HF) calculations for electrons in a parabolic quantum dot at zero magnetic field. The crossover from Fermi liquid to Wigner molecule behavior is studied for up to eight electrons and various spin components $S_{z}$. We compare the results with numerically exact path-integral Monte Carlo simulations and earlier HF studies. Even in the strongly correlated regime the symmetry breaking HF solutions provide accurate estimates for the energies and describe the one-particle densities qualitatively. However, the HF approximation favors the formation of a Wigner molecule and produces azimuthal modulations of the density for even numbers of electrons in one spatial shell.

PACS numbers: 73.20.Dx, 71.10Ay, 71.10.Hf

The last decade has seen an enormous interest in quantum dots, i.e., a small number of $2 \mathrm{D}$ electrons confined in a semiconductor heterostructure [1]. Experimentally, the $N$-electron states of these systems are studied by means of far-infrared [2], capacitance [3] and transport spectroscopy 沺, and exhibit features of quantization of charge and energy. Theoretically, a whole arsenal of methods for interacting electronic systems together with increasing computational power applies: Exact diagonalization techniques [5], density functional theory [6] and quantum Monte Carlo methods [7]. Recently, also the very strongly correlated regime of small electronic densities has attracted considerable interest [8 10]. In particular, the formation of a Wigner molecule was studied with Quantum Monte Carlo (QMC) [8], Hartree-Fock (HF) [9] and configuration interaction [10] calculations.

In this paper we reconsider the Hartree-Fock approximation focusing on the crossover to the Wigner regime. An important practical and conceptual question to be clarified is how correlations beyond the mean field approximation contribute to the exact energy and whether the Wigner molecule is described reliably within HF. In its unrestricted version, allowing for symmetry-broken solutions, HF approaches the true ground state energy considerably better than restricted HF which preserves the rotational symmetry of the Hamiltonian. This is achieved, however, on expense of the quality of the wave functions.

In the case of a strong central potential in three dimensions, such as in real atoms, the HF approximation is known to yield useful results both for energies and wave functions. Here, we show by comparison with exact Monte Carlo data [8] that even for strong interaction un- restricted HF can give very good estimates for the ground state energies of 2D quantum dots. Only tiny energy differences between different spin states cannot be resolved reliably. On the other hand, the charge density distribution resulting from unrestricted $\mathrm{HF}$ cannot quantitatively describe the strongly correlated regime. For even number of electrons per shell, the HF densities show effects of localization due to the strong electron interaction. Furthermore, within HF [9] this crystallization sets in rather too early at higher densities than in the QMC study [8].

Model - We study a two-dimensional parabolic quantum dot with $N$ electrons at zero magnetic field as it has been discussed by many authors [5-10]. Measuring energy in units of the oscillator energy $\hbar \omega_{0}$ and length in units of $l_{0}=\sqrt{\hbar / m^{*} \omega_{0}}$, where $m^{*}$ is the effective mass, the dimensionless Hamiltonian reads

$$
\begin{aligned}
H & =\sum_{j=1}^{N}\left(-\frac{1}{2} \Delta_{j}+\frac{1}{2} \boldsymbol{r}_{j}^{2}\right)+\sum_{i<j=1}^{N} \frac{\lambda}{\left|\boldsymbol{r}_{i}-\boldsymbol{r}_{j}\right|} \\
& \equiv \sum_{i=1}^{N} h_{i}+\sum_{i<j=1}^{N} w_{i j} .
\end{aligned}
$$

Here we have introduced the dimensionless coupling constant $\lambda=l_{0} / a_{\mathrm{B}}^{*}=e^{2} / \kappa l_{0} \hbar \omega_{0}$ with the effective Bohr radius $a_{\mathrm{B}}^{*}$ and the dielectric constant $\kappa$. For example $\lambda=2$ corresponds to $\hbar \omega_{0} \approx 3 \mathrm{meV}$ for a GaAs quantum dot. Since this Hamiltonian is rotationally invariant and spin independent, the exact eigenfunctions can be chosen as simultaneous eigenfunctions of the total angular momentum $L_{z}^{\text {tot }}$, the total spin $\boldsymbol{S}_{\text {tot }}^{2}$ and its $z$-component $S_{z}^{\text {tot }}$. These eigenfunctions and corresponding densities are then rotationally invariant.

Method - HF theory consists in approximating the many-particle wave function by an optimal single Slater determinant

$$
\Psi^{\mathrm{HF}}=\frac{1}{\sqrt{N !}} \operatorname{det}\left(\varphi_{i}\left(\boldsymbol{r}_{j}\right)\right)_{1 \leq i, j \leq N} .
$$

This HF Slater determinant is build up by single particle orbitals $\varphi_{i}(\boldsymbol{r})$, which are expanded in the angular momentum basis of the 2D harmonic oscillator (FockDarwin states)

$$
\langle\boldsymbol{r} \mid n M\rangle=\sqrt{\frac{n !}{\pi(n+|M|) !}} e^{i M \varphi} r^{|M|} \mathcal{L}_{n}^{|M|}\left(r^{2}\right) e^{-r^{2} / 2} .
$$


Here, $n$ and $M$ are the radial and angular quantum numbers, and $\mathcal{L}_{n}^{|M|}$ is a Laguerre polynomial. In the unrestricted HF approximation an orbital has the expansion

$$
\varphi_{i}(\boldsymbol{r})=\sum_{\substack{n=0, \infty \\ M=-\infty, \infty}} u_{n M}^{i}\left\langle\boldsymbol{r} \mid n M \sigma_{i}\right\rangle
$$

where $\sigma_{i}= \pm \frac{1}{2}$ is the fixed electron spin of the $i$-th orbital. This orbital is no longer an eigenfunction of the one particle angular momentum, therefore the HF Slater determinant is in general not an eigenfunction of $L_{z}^{\text {tot }}$, but a deformed, symmetry broken solution [11]. Similarly, the HF solution is in general not an eigenfunction of the total spin but only of its $z$-component with eigenvalue $S_{z}^{\text {tot }} \equiv S_{z}=\left(N_{\uparrow}-N_{\downarrow}\right) / 2=\sum_{i} \sigma_{i}$.

Minimizing the $\mathrm{HF}$ energy $E^{\mathrm{HF}}=\left\langle\Psi^{\mathrm{HF}}|H| \Psi^{\mathrm{HF}}\right\rangle$ and imposing orthonormality between the HF orbitals yields the HF equations for the expansion coefficients $u_{n M}^{i}$

$$
\sum_{\alpha}\left\{\langle\gamma|h| \alpha\rangle+\sum_{\beta \beta^{\prime}}\left(\gamma \beta|w| \alpha \beta^{\prime}\right) \rho_{\beta^{\prime} \beta}\right\} u_{\alpha}^{k}=\varepsilon_{k} u_{\gamma}^{k} .
$$

Here, greek indices abbreviate the quantum numbers of (3), for example $\alpha \equiv(n, M, \sigma)$. The density matrix is defined by $\rho_{\alpha \alpha^{\prime}}=\sum_{i=1}^{N} u_{\alpha}^{i} u_{\alpha^{\prime}}^{i},\langle\gamma|h| \alpha\rangle$ is the one particle matrix element and $\left(\alpha \alpha^{\prime}|w| \beta \beta^{\prime}\right)=\left\langle\alpha \alpha^{\prime}|w| \beta \beta^{\prime}\right\rangle-$ $\left\langle\alpha \alpha^{\prime}|w| \beta^{\prime} \beta\right\rangle$ denotes the antisymmetrized Coulomb matrix element, which we calculated analytically by transformation into relative and center of mass coordinates [13]. The nonlinear selfconsistent eigenvalue problem (5) for the expansion coefficients $u_{\alpha}^{i}$ is solved by iterative diagonalization starting from an initial guess for the density matrix. Several starting guesses have to be chosen in order to avoid local minima. The true minimum can also be identified by its one particle density,

$$
n^{\mathrm{HF}}(\boldsymbol{r})=\sum_{i=1}^{N}\left|\varphi_{i}(\boldsymbol{r})\right|^{2},
$$

which in the strong coupling limit mirrors the geometry of classical electrostatic point-charges as we discuss below.

In our calculations we used up to 55 Fock-Darwin states for each spin direction. We present results for various sets of $N, \lambda, S_{z}$ from $N=2$ to 8 particles covering the whole range of interaction strengths from $\lambda=2$ to 10.

HF energies and spin - The most important properties of the HF ground state energies can already be seen for $N=2$ (quantum dot Helium). Here one knows the exact solution from diagonalization of the Hamiltonian for the relative motion [5]. We have reproduced these results in Table If The true ground state is always a singlet and the energy gap to the triplet vanishes slowly as $\lambda \rightarrow \infty$. In contrast, the HF approximation finds a triplet ground state for $\lambda \gtrsim 5$, and the energies of singlet and triplet converge more rapidly [12]. The (absolute and relative) error is largest in the case of $S_{z}=0$ and $\lambda \approx 2$. In the polarized case the error of HF increases monotonically with $\lambda$. These features of the HF energies can be seen for all $N$.

For $N>2$ we compare with the path integral QMC data of Ref. [8], which have been obtained for the very low temperature $T=0.1 \hbar \omega_{0} / k_{\mathrm{B}}$. The HF ground state energies are always above the QMC energies for all particle numbers investigated so that the QMC data may serve effectively as zero temperature reference points. In the case of $N=5$ electrons, QMC gives a ground state spin of $1 / 2$. In Fig. 1 we plot the energy difference of the different spin states with respect to the true ground state, $\Delta E=E_{S}-E_{1 / 2}^{\mathrm{QMC}}$, for various spin states as a function of the coupling strength $\lambda$. Again we see that $\Delta E$ is largest for $\lambda \approx 2$ and smallest spin. It remains constant for $\lambda \gtrsim 4$, resulting in a very low relative error of only $1-2 \%$ in the low density regime. The HF energy is very accurate in the case of $S_{z}=5 / 2$ which becomes the HF ground state for $\lambda \gtrsim 4$. This unphysically high ground state spin is due to the exchange term in the HF energy, which lowers the energy only for parallel spins. Therefore the spin ordering of the HF energies is just reversed.

For $N=8$ we give the HF energies in Table III. The QMC predicts a transition of the ground state spin from $S=1$ to $S=2$ for $\lambda \gtrsim 4$. Again HF finds the wrong spin ordering in the correlated regime. The relative error is largest for the unpolarized states. The convergence of the HF energies for different $S_{z}$ for $\lambda \gtrsim 6$ can be understood in the classical picture of localized electrons without overlap and therefore no spin sensitivity.

HF densities - Next we study the HF one particle densities (6), where the crossover to the Wigner molecule is discernible. However, one has to keep in mind that the deformation and structure of the HF densities arise from the symmetry violating mean field and therefore may be artificial (whereas the HF energies are a true upper bounds for the ground state energies). Second we want to point out that a symmetry breaking Slater determinant does not necessarily mean that its corresponding density displays a molecule-like structure. In Fig. 2 we display the densities for $N=2$ to 5 for strong coupling $\lambda=6$ and maximal spin $S_{z}=N / 2$ (From $\lambda \gtrsim 6$ the HF densities are essentially the same for all spins.). In the case of 2 and 4 electrons they show quite distinct azimuthal maxima. This is not the case for the odd electron numbers 3 and 5: Surprisingly the HF densities, though belonging to a deformed Slater determinant, seem to be rotationally symmetric.

In order to understand this even-odd effect consider an exact spin-polarized $N$-electron wave function $\Psi_{N}$ for the Wigner molecule case. An even number of electrons in one spatial shell carries a nonzero angular momen- 
tum $\pm \hbar N / 2$ 14. In this fashion the HF wavefunctions with modulated density $(N=2,4)$ can be interpreted as standing waves from a superposition of opposite angular momenta [15].

However the HF densities display the right classical filling for the spatial shells: From the maxima in the densities one can also read off the Brueckner parameter $r_{s}$, which is defined as the the nearest-neighbor distance in units of $a_{\mathrm{B}}^{*}$. These values $r_{s}$ agree well with those obtained by QMC and from a model of classical pointcharges. In Fig. 3 we show a 7-electron Wigner molecule for two interaction strengths. For $\lambda=2$ the HF density is already 6 -fold modulated with a central electron. Confronting this with the exact density in Fig. 1 of Ref. [8], we notice that the crystallization (as suggested by the HF densities) occurs too early. This explains why the authors of Ref. [9] observe Wigner crystallization for higher densities than in the exact QMC study 8]. For higher $\lambda$ the molecule with two spatial shells becomes more and more distinct. The maxima agree very well with the classical formula $r_{s}^{3}=\lambda^{4}(2.25+1 / \sqrt{3})$ for the 6 -fold geometry.

Finally we show in Fig. 4 the crystallization for $N=6$ and $S_{z}=0$. This unpolarized case depicts a crossover from a 6 -fold arrangement [Fig. $4($ a $)$ ] to a 5-fold geometry [Fig. $4(\mathrm{c})$ and $(\mathrm{d})]$. The 6-electron molecule was also studied by Reimann et al. [10], who found by means of configuration interaction calculations that the true ground state was unpolarized with a 6 -fold symmetry up to at least $\lambda \approx 3.5$. Within $\mathrm{HF}$ the $S_{z}=0$ state acquires a 5 -fold symmetry already for $\lambda \geq 2.85$. In this range the $\mathrm{HF}$ density is distorted $[\lambda=4$ in Fig. $4(\mathrm{c})]$ and then again apparently round for higher lambda $[\lambda=6$ in Fig. $4(d)$ ] with a central maximum. Fig. $4(b)$ shows the 6 -fold isomer for $\lambda=4$, which is by 0.33 higher in energy than the ground state in Fig. A(c). In contrast the spinpolarized state exhibits 5-fold symmetry throughout the whole parameter range [16].

In conclusion we have shown in how far an unrestricted HF description of the Wigner molecule in quantum dots is reliable. The HF energies are good estimates of the true ground state energies especially for spin-polarized states. The energy differences for different spin-states in the strong interacting regime cannot be resolved properly. We find deformed $\mathrm{HF}$ densities in the regime of intermediate interaction up to $\lambda \lesssim 4$. For strong correlation the densities are azimuthally modulated for an even number of electrons in a shell and round for an odd number per shell. The onset of this modulation is enhanced within $\mathrm{HF}$, which leads to overestimate the value of the critical density for the crossover to the Wigner molecule. However the HF densities mirror the classical filling scheme with the electrons arranged in spatial shells.

We thank R. Blümel, R. Egger, C. Stafford, and T. Vorrath for useful discussions. This research has been supported by the SFB 276 of the Deutsche Forschungs- gemeinschaft (Bonn)

[1] L. Jacak, P. Hawrylak, and A. Wójs, Quantum Dots (Springer-Verlag, Heidelberg, 1998).

[2] B. Meurer, D. Heitmann, and K. Ploog, Phys. Rev. Lett. 68, 1371 (1992).

[3] R. Ashoori, Nature 379, 413 (1996).

[4] L. P. Kouvenhouwen et al., in Mesoscopic Electron Transport, Vol. 354 of NATO-ASI Series E, edited by L. L. Sohn, L. P. Kouvenhouwen, and G. Schön (Kluwer Academic Publishers, Dordrecht, 1997), pp. 105-214.

[5] U. Merkt, J. Huser, and M. Wagner, Phys Rev. B 43, 7320 (1991).

[6] K. Hirose and N. S. Wingreen, Phys. Rev. B 59, 4604 (1999).

[7] J. Harting, O. Mülken, and P. Borrmann, condmat/0002269.

[8] R. Egger, W. Häusler, C. H. Mak, and H. Grabert, Phys. Rev. Lett. 82, 3320 (1999), 83, 462(E) (1999).

[9] C. Yannouleas and U. Landman, Phys. Rev. Lett. 82, 5325 (1999).

[10] S. M. Reimann, M. Koskinen, and M. Maninen, condmat/0004093.

[11] We also performed restricted $\mathrm{HF}$ calculations where the angular momentum of $\varphi_{i}$ is fixed.

[12] In the restricted $\mathrm{HF}$ the energies for different $S_{z}$ do not merge, resulting in a considerably larger error for the unpolarized states.

[13] B. Reusch, Diploma thesis, University of Freiburg, 1998.

[14] A. J. Leggett in Granular Nanoelectronics, ed. by D. K. Ferry, J. R. Barker, and C. Jacoboni, NATO ASI Ser. B, Vol. 251, Plenum New York 1991.

[15] A corresponding effect for weak interaction was observed in the density-functional study of Ref. [6].

[16] We were not able to reproduce the density of Fig. 2(c) in Ref. [9] unless we introduce a magnetic field (then we recover the results of H.-M. Müller and S. E. Koonin, Phys. Rev. B 54, 14532 (1996)).

TABLE I. Lowest energies of exact diagonalization and HF for $N=2$ and various $S_{z}$ and coupling strengths $\lambda$. Relative error $\left(E_{S}^{\text {exact }}-E_{S}^{\text {exact }}\right) / E_{S}^{\text {exact }}$ in $\%$.

\begin{tabular}{|lcccc|}
\hline \hline$\lambda$ & $S_{z}$ & $E^{\text {exact }}$ & $E^{\mathrm{HF}}$ & rel. err. \\
\hline 2 & 1 & 4.142 & 4.168 & 0.7 \\
2 & 0 & 3.721 & 4.034 & 8.3 \\
\hline 4 & 1 & 5.119 & 5.189 & 1.4 \\
4 & 0 & 4.848 & 5.182 & 6.8 \\
\hline 6 & 1 & 5.990 & 6.096 & 1.8 \\
6 & 0 & 5.784 & 6.107 & 5.7 \\
\hline 8 & 1 & 6.787 & 6.919 & 1.9 \\
8 & 0 & 6.618 & 6.930 & 4.7 \\
\hline 10 & 1 & 7.528 & 7.679 & 2.0 \\
10 & 0 & 7.384 & 7.686 & 4.2 \\
\hline \hline
\end{tabular}




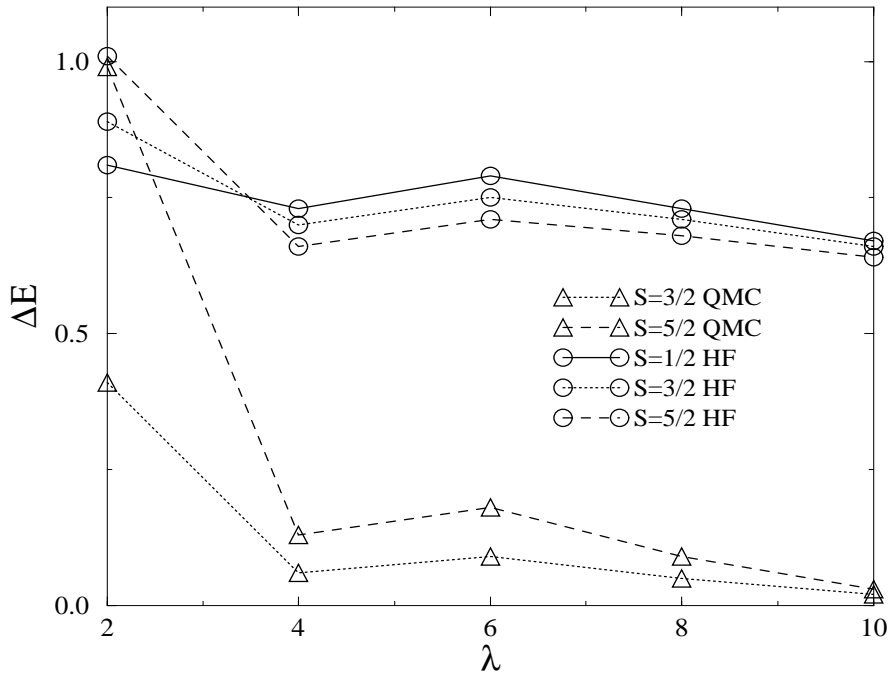

FIG. 1. $N=5$. Absolute energy differences to QMC ground state $(S=1 / 2), \Delta E=E_{S}-E_{\mathrm{GS}}^{\mathrm{QMC}}$, for various spins vs. coupling constant $\lambda$
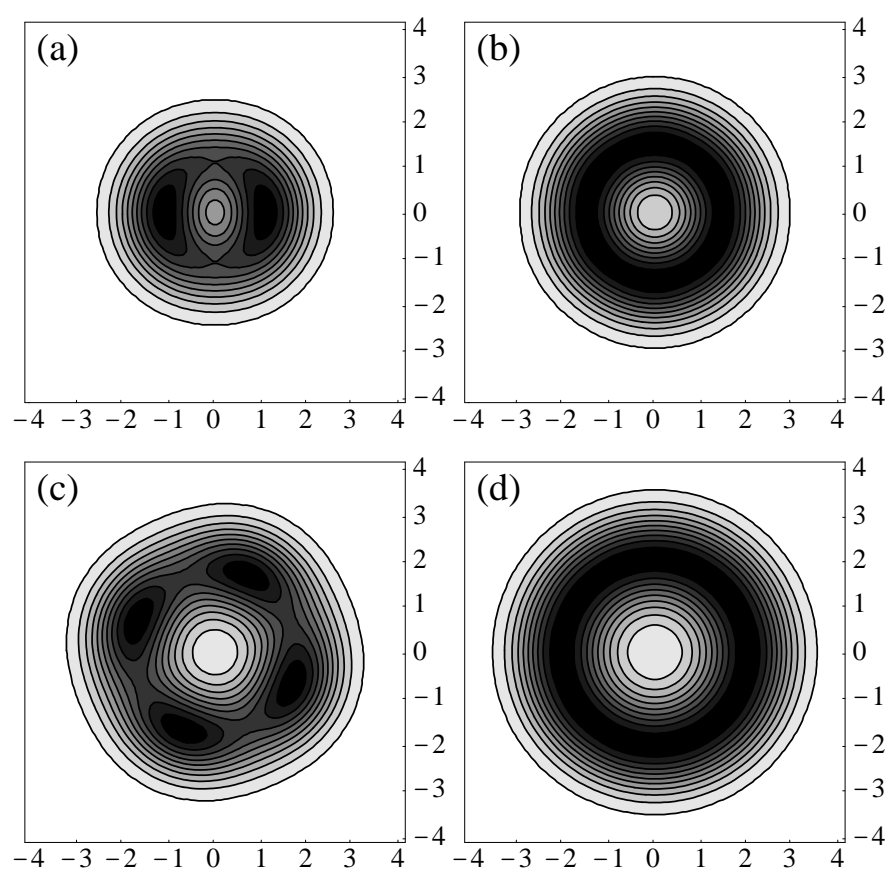

FIG. 2. Shadowed contour-plot of the HF density $n^{\mathrm{HF}}$ for $\lambda=6, S_{z}=N / 2$ and different electron numbers. Contours lie at integral multiples of 0.1 times the maximal density. (a) $N=2$, (b) $N=3$, (c) $N=4$, (d) $N=5$.

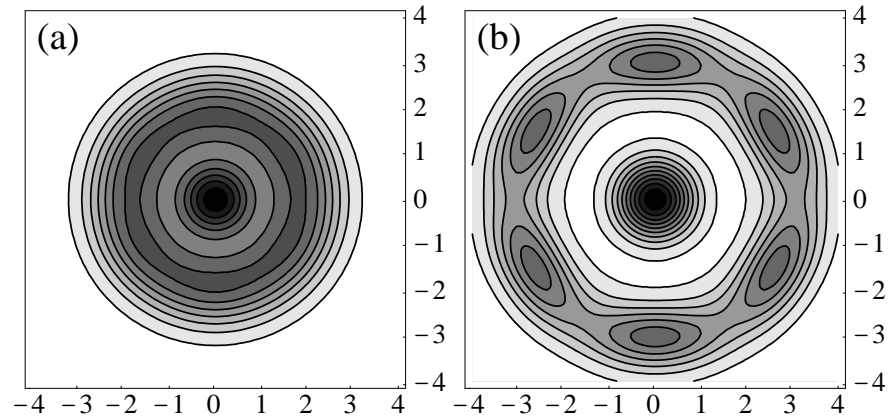

FIG. 3. 7-electron Wigner molecule. HF density $n^{\mathrm{HF}}$ for (a) $\lambda=2 S_{z}=1 / 2$, (b) $\lambda=10 S_{z}=7 / 2$.
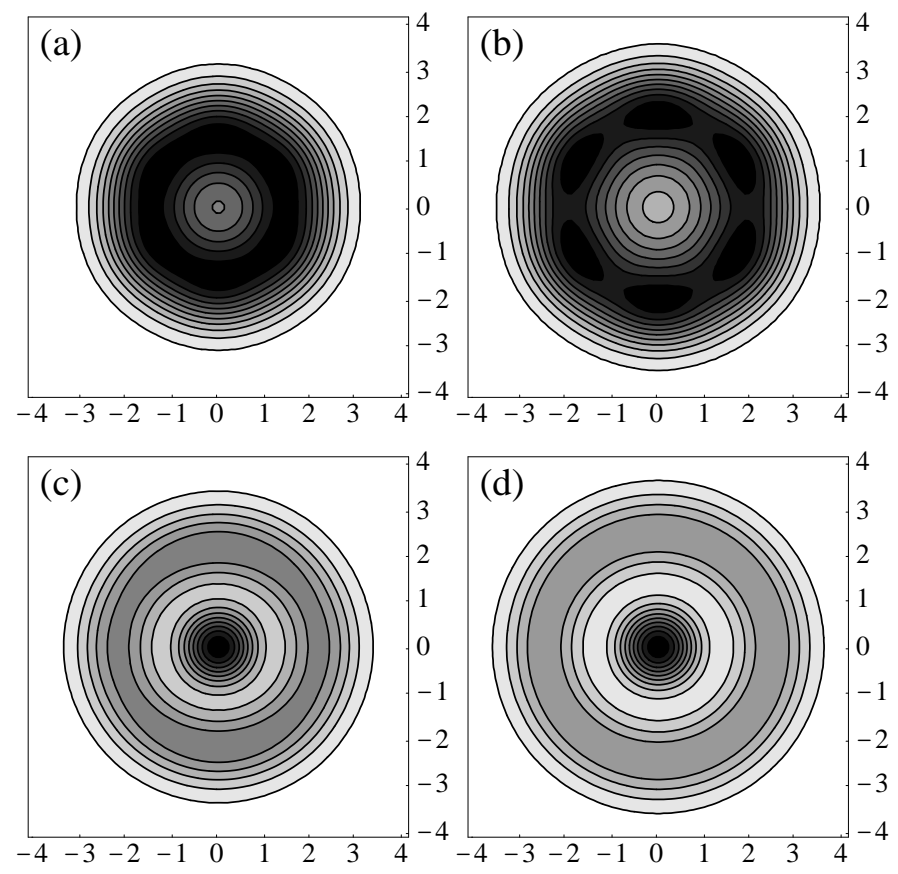

FIG. 4. $N=6 S_{z}=0$. Rearrangement from a 6-fold [(a) $\lambda=2]$ to a (deformed) 5 -fold geometry [(c) $\lambda=4$, $E=41.509$, (d) $\lambda=6]$. In (b) 6 -fold isomer at $\lambda=4$ with energy $E^{*}=41.838$. 
TABLE II. Energies of QMC vs. HF for $N=8$. Bracketed numbers denote statistical errors of QMC. Relative error $\left(E_{S}^{\mathrm{UHF}}-E_{S}^{\mathrm{QMC}}\right) / E_{S}^{\mathrm{QMC}}$ in $\%$.

\begin{tabular}{|lcccc}
\hline \hline$\lambda$ & $S_{z}$ & $E^{\mathrm{QMC}}$ & $E^{\mathrm{HF}}$ & rel. err. \\
\hline 2 & 4 & $48.3(2)$ & 48.534 & 0.5 \\
2 & 3 & $47.4(3)$ & 48.336 & 2.0 \\
2 & 2 & $46.9(3)$ & 48.243 & 2.9 \\
2 & 1 & $46.5(2)$ & 48.132 & 3.5 \\
\hline 4 & 4 & $69.2(1)$ & 69.735 & 0.8 \\
4 & 3 & $68.5(2)$ & 69.783 & 1.9 \\
4 & 2 & $68.3(2)$ & 69.826 & 2.2 \\
\hline 6 & 4 & $86.92(6)$ & 87.957 & 1.2 \\
6 & 3 & $86.82(5)$ & 87.999 & 1.4 \\
6 & 2 & $86.74(4)$ & 88.039 & 1.5 \\
\hline 8 & 4 & $103.26(5)$ & 104.492 & 1.2 \\
8 & 3 & $103.19(4)$ & 104.520 & 1.3 \\
8 & 2 & $103.08(4)$ & 104.547 & 1.4 \\
\hline \hline
\end{tabular}

